\title{
A Computational Approach Using Bioinformatics to Screening Drug Targets for Leishmania infantum Species
}

\author{
Miguel Angel Chávez-Fumagalli, ${ }^{1}$ Mônica Santos Schneider, \\ Daniela Pagliara Lage ${ }^{D}{ }^{1},{ }^{1}$ Grasiele de Sousa Vieira Tavares, ${ }^{1}$ \\ Débora Vasconcelos Costa Mendonça, ${ }^{1}$ Thaís Teodoro de Oliveira Santos, ${ }^{1}$ \\ Rodrigo Maia Pádua, ${ }^{2}$ Ricardo Andrez Machado-de-Ávila, ${ }^{3}$ \\ João Paulo Viana Leite $\mathbb{D},{ }^{4}$ and Eduardo Antonio Ferraz Coelho $\mathbb{D}$ \\ ${ }^{1}$ Programa de Pós-Graduação em Ciências da Saúde: Infectologia e Medicina Tropical, Faculdade de Medicina, \\ Universidade Federal de Minas Gerais, Belo Horizonte, MG, Brazil \\ ${ }^{2}$ Departamento de Produtos Farmacêuticos, Faculdade de Farmácia, Universidade Federal de Minas Gerais, \\ 31.270-901 Belo Horizonte, MG, Brazil \\ ${ }^{3}$ Programa de Pós-Graduação em Ciências da Saúde, Universidade do Extremo Sul Catarinense, Criciúma, SC, Brazil \\ ${ }^{4}$ Departamento de Bioquímica e Biologia Molecular, Universidade Federal de Viçosa, 36.570-000 Viçosa, MG, Brazil
}

Correspondence should be addressed to Eduardo Antonio Ferraz Coelho; eduardoferrazcoelho@yahoo.com.br

Received 29 August 2017; Accepted 25 February 2018; Published 28 March 2018

Academic Editor: Nunziatina De Tommasi

Copyright (C) 2018 Miguel Angel Chávez-Fumagalli et al. This is an open access article distributed under the Creative Commons Attribution License, which permits unrestricted use, distribution, and reproduction in any medium, provided the original work is properly cited.

\begin{abstract}
Background. The development of new therapeutic strategies to treat patients for leishmaniasis has become a priority. The antileishmanial activity of the strychnobiflavone flavonoid was recently demonstrated against Leishmania amazonensis and Leishmania infantum amastigotes and promastigotes. The biological effect of this molecule was identified due to its capacity to interfere in the parasite mitochondrial membrane; however, the underlying molecular mechanism remains unclear. Methods and Results. In this study, a computational approach using bioinformatics was performed to screen biological targets of strychnobiflavone in L. infantum. Computational programs, such as the target fishing approach and molecular docking assays, were used. Results showed that the putative pathway targeted by strychnobiflavone in L. infantum is the methylglyoxal degradation superpathway, and one hydrolase-like protein was predicted to be the molecular target of this flavonoid in the parasites. Conclusion. In this context, this study provides the basis for understanding the mechanism of action of strychnobiflavone in L. infantum and presents a strategy based on bioinformatics programs to screen targets of other molecules with biological action against distinct pathogens.
\end{abstract}

\section{Introduction}

Visceral leishmaniasis (VL) is a potentially fatal disease caused by the protozoan Leishmania infantum found throughout the Mediterranean, Southwest Asia, China, Central America, and South America [1]. The parasites are transmitted by the bite of infected phlebotomine sand flies and can parasitize mammalian cells in organs, such as the hosts' spleen, bone marrow, and liver [2,3]. The clinical manifestations of the disease vary from an asymptomatic infection to fatal visceral disease [4-6]. The parenteral administration of pentavalent antimonials continues to be the first choice as VL treatment; however, the occurrence of side effects, such as myalgias, arthralgias, chemical pancreatitis, and cardiotoxicity, has also been identified in patients [7].

Amphotericin $\mathrm{B}$ is an antifungal drug presenting antileishmanial activity; however, its clinical use is limited by the high toxicity and/or high cost of lipid-based formulations [8-10]. As a consequence, the search for new treatment products for VL is considered as a priority [11]. A number 
of natural product-derived compounds have shown a significant role against different diseases $[12,13]$. Over the past decade, about 340 natural compounds were identified as having promising antileishmanial activity [14].

In this context, greater attention has been given to plants evaluation, seeking to identify new antileishmanial products $[15,16]$. Plants present secondary products resulting from their metabolism, with well-defined chemical structures, representing a basis for new pharmaceuticals [17]. In addition, the wide variety of modern techniques of purification has allowed for the identification of new compounds that can in turn become effective antileishmanial products [18].

Recently, an ethyl acetate extract derived from Strychnos pseudoquina stem bark proved to be effective against different Leishmania species. Two flavonoids, quercetin 3-O-methyl ether and strychnobiflavone, were identified as the main responsible agents for this antileishmanial activity [19]. These molecules presented low toxicity in murine macrophages and a null hemolytic activity in human red blood cells. In a new study, the mechanism of action of strychnobiflavone in $L$. infantum proved to be related to alterations induced by this molecule in the parasite's mitochondrial membrane potential [20].

Aiming to screen the molecular target of this flavonoid in L. infantum by means of distinct bioinformatics programs, the present study applied a computational approach based on target fishing and molecular docking assay. Moreover, investigations of drug-drug and drug-human protein interactions were developed to evaluate the interactive mechanisms of this molecule with mammalian proteins, which could eventually cause adverse effects in the patients during antileishmanial treatment.

\section{Material and Methods}

2.1. Target Fishing Approach. Target fishing screen was based on chemical similarity, as well as on the use of current knowledge of the bioactivity of small molecules [21]. These methodologies were based on the "chemical similarity principle," in which similar molecules are likely to have equivalent properties [22]. For this, the chemical structure of strychnobiflavone was retrieved from the PubChem database [23] and uploaded to the TargetHunter [24], SwissTargetPrediction [25], Similarity Ensemble Approach (SEA) [26], and PASS Online [27] servers. Threshold values were selected by default parameters, and molecular targets were considered as possible "hits," when the four algorithms presented a consensual result.

2.2. Literature Review. Since the main tools offered by the servers to evaluate target fishing are related to human proteins, a cross-reference with $L$. infantum-related proteins was performed. For this, "hits" were employed as keywords in a literature review performed on the PubMed server (https://www.ncbi.nlm.nih.gov/pubmed), as described in [28]. Next, the obtained data were manually extracted, information about L. infantum metabolic pathways was retrieved from the Kyoto Encyclopedia of Genes and Genomes (KEGG) database [29], and a manual comparison was performed. The complete sequence-based pathway analysis of the information was retrieved from MetaCyc [30].

2.3. Protein-Protein Interaction Search. The proteins belonging to the predicted metabolic pathway were chosen to analyze their interaction with other molecules. For this, the Retrieval of Interacting Genes (STRING) program was employed. This server contains known and unknown protein associations, based not only on the direct and physical association of proteins, but also on their genetic interactions and involvement in subsequent catalysis steps in the metabolic processes [31]. All obtained sequences were selected for further analysis, and their FASTA sequences were retrieved from the UniProt database (http://www.uniprot.org/), using their identification numbers.

2.4. Protein Sequence Comparison. The L. infantum protein sequences obtained by using the STRING server were subjected to BLAST assay [32], and the sequence's similarity search was performed by using murine and human databases. The "expect" value ( $e$-value) was lower than 0.005 , and a minimum hit score higher than 100.0 was used to exclude homologous sequences. The proteins that showed hits with the aforementioned cut-off values were considered to be "nonhomologous" proteins [33-35] and were used in the subsequent analyses, while remaining sequences were excluded.

2.5. Homology Modeling. The amino acid sequences of the selected proteins were uploaded in a FASTA format to the Iterative Threading Assembly Refinement (I-TASSER) server. Tertiary structures were predicted in PDB format, and results showed five top models for each entry, where ones with the highest confidence score (c-score) represented the best model [36].

2.6. Druggable Pocket Identification. The active sites in the evaluated tertiary structures of selected proteins were identified by using the DoGSiteScorer server [37], in which the druggability of a pocket can be automatically predicted through the analyses of its size, shape, and chemical features. Considering all descriptors, the DoGSiteScorer server provides a drug score value (0-1) for a selected pocket, where a higher score and a druggable pocket were estimated.

2.7. Molecular Docking Assay. The tertiary structures predicted by the I-TASSER server were used to perform a docking assay in the strychnobiflavone structure by using the SwissDock server [38]. Binding modes were scored using their FullFitness and clustered. Clusters were ranked according to the average FullFitness of their elements, and results of the SwissDock were viewed using the UCSF Chimera package [39].

2.8. Functional Annotation of Hypothetical Proteins. The experimental strategy was developed as described in [40]. Briefly, the functional domain of selected proteins was evaluated by the following programs: Pfam [41], PANTHER 10.0 [42], SUPERFAMILY [43], SMART [44], CATH [45], 
and ProtoNet 6.0 [46]. The Receiver Operator Characteristic (ROC) curves were constructed to estimate the protein localization and function in the parasite. Results were expressed as sensitivity (Se), specificity (Sp), accuracy (Ac), and area under the curve (AUC).

2.9. Chemical-Protein Interactome Profile of Strychnobiflavone. The chemical-protein interactome (CPI) refers to the information of interaction of a panel of chemicals across target proteins, in terms of binding strength and conformation to each chemical-protein pocket pair [47]. Both DRARCPI and DDI-CPI servers are employed for computational drug repositioning by the CPI server $[48,49]$. The molecular structure of strychnobiflavone was submitted to the DRARCPI and DDI-CPI servers, and parameters were set to the default values. Results were considered satisfactory when the algorithms presented positive consensual data.

\section{Results}

3.1. Target Fishing Approach. The molecular structure of strychnobiflavone was analyzed by distinct bioinformatics programs, aiming to screen the metabolic pathway of this molecule on L. infantum, as well as its molecular target in these parasites. For this, the structure of the flavonoid was evaluated by applying distinct algorithms, which used chemical similarity to identify proteins with known ligands to show similarity to this molecule [50]. In the results, the TargetHunter, Swiss TargetPrediction, SEA, and PASS servers identified 21, 15, 75, and 630 putative targets, respectively. A positive consensual result was obtained with three hits: NADPH oxidase, Aldose reductase, and Aldo-keto reductase, which were employed as keywords for a literature review. The aim was to perform an evaluation of cross-reference between these terms and Leishmania proteins, as well as to search for references about their involvement in the parasite's biology. The following strategies were entered in the PubMed server: ("NADPH oxidase" [MeSH Terms] OR ("NADPH" [All Fields] AND “oxidase" [All Fields]) OR "NADPH oxidase" [All Fields]) AND ("leishmanial" [MeSH Terms] OR "leishmanial" [All Fields]) for ["NADPH oxidase"], resulting in 35 references founded; and ("aldehyde reductase" [MeSH Terms] OR ("aldehyde" [All Fields] AND "reductase" [All Fields]) OR “aldehyde reductase" [All Fields] OR ("aldose" [All Fields] AND "reductase" [All Fields]) OR “aldose reductase" [All Fields]) AND ("leishmanial” [MeSH Terms] OR "leishmanial" [All Fields]) for ["Aldose reductase"], resulting in eight identified references. In the case of ["Aldoketo reductase"], only one reference was found. Data were extracted, analyzed, and compared with the metabolic pathway information present in the KEGG and MetaCyc servers. The results showed that the mechanism of action of strychnobiflavone was based on the inhibition of the methylglyoxal degradation superpathway (Figure 1).

3.2. STRING Analysis and Sequence Homology. The distribution of Glyoxalase system proteins in L. infantum showed the presence of Glyoxalase I (EC 4.4.1.5), Glyoxalase II (EC 3.1.2.6), and Aldo-keto reductase (EC 1.1.1.21) proteins [51].
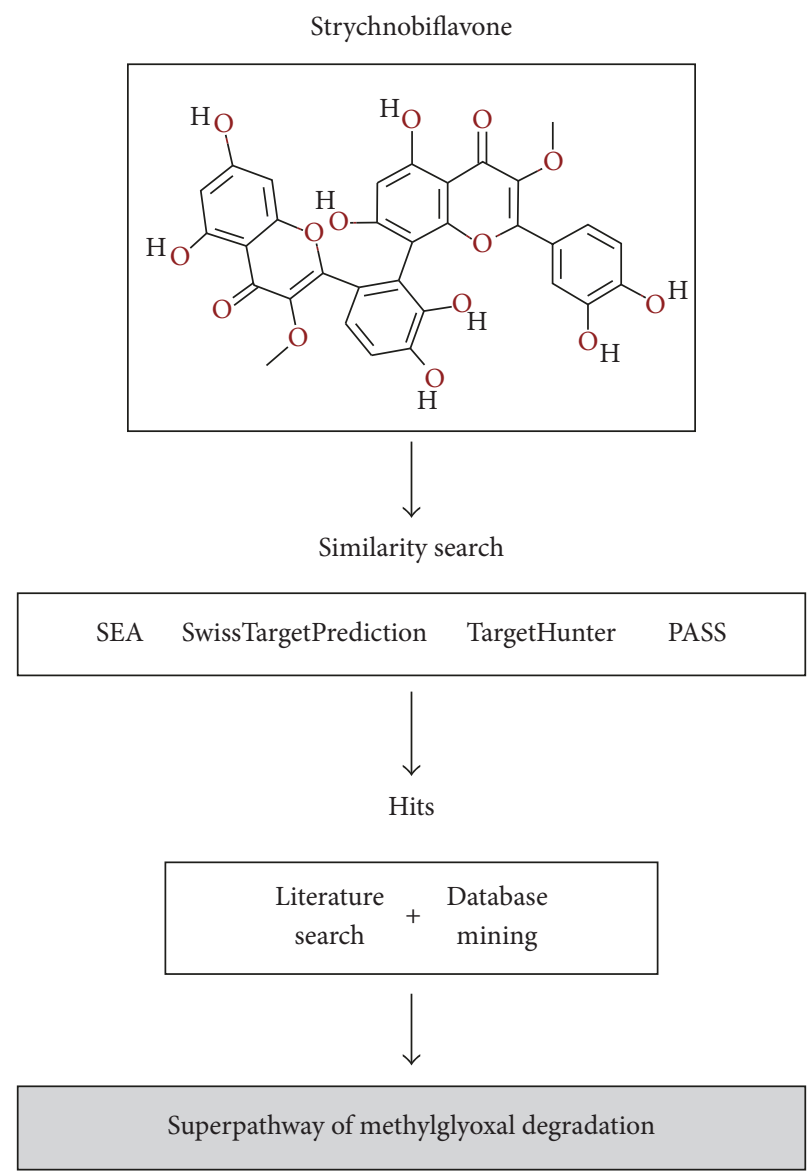

FIGURE 1: Computational framework used in the target fishing prediction of strychnobiflavone in the Leishmania infantum metabolic superpathway.

The amino acid sequences of these antigens were submitted to a STRING analysis, and nine sequences were identified to interact with Glyoxalase I or Glyoxalase II proteins, whereas 10 sequences were identified to interact with Aldo-keto reductase. Since strychnobiflavone presents low toxicity in mammalian cells [19], one could speculate that its target is absent or expressed in low levels in these cells. Next, a homology analysis against human proteins was performed, and six sequences related to the Glyoxalase proteins were selected by their significant distinction with their homolog in mammalians (Table 1). These amino acid sequences were then selected for further analysis.

3.3. Molecular Modeling, Druggability, and Docking Assay. The structural prediction of a protein is performed by means of bioinformatics programs and theoretical chemistry, which is required, given that protein functions are dependent on their defined chemical structure [52]. In this sense, the six previously selected sequences were submitted to an automated homology model using the I-TASSER server, and, based on the $c$-scores, the best model was selected (Table 2). In addition, binding sites were detected in the screening models and were analyzed in terms of both their 


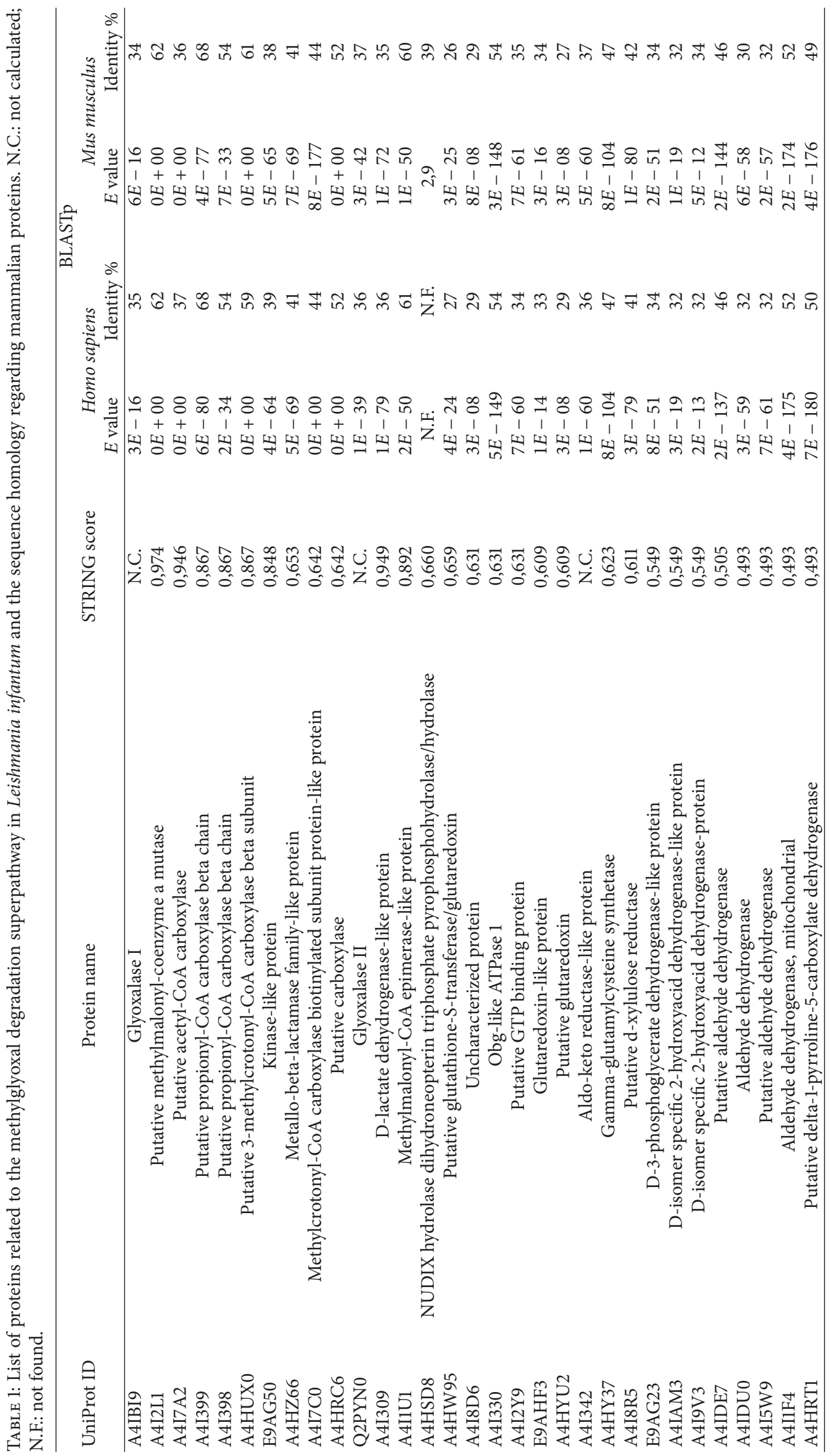


TABLE 2: List of selected proteins and their evaluation as putative molecular targets of strychnobiflavone.

\begin{tabular}{lcccccc}
\hline \multirow{2}{*}{ UniProt ID } & \multicolumn{2}{c}{ Molecular Modelling } & \multicolumn{2}{c}{ Druggability } & \multicolumn{2}{c}{ Molecular docking } \\
& PDB hit & C-score & Drug score & Simple score & FullFitness (kcal/mol) & Estimated $\Delta G(\mathrm{kcal} / \mathrm{mol})$ \\
\hline A4IBI9 & 2c21A & 1.27 & 0.58 & 0.20 & -498.92 & -8.03 \\
A4HSD8 & 2kdvA & -0.65 & 0.75 & 0.28 & -501.41 & -8.37 \\
A4I8D6 & 4r04A & -0.82 & 0.81 & 0.62 & -2985.25 & -8.67 \\
E9AHF3 & 3h8qA & 0.26 & 0.73 & 0.08 & -244.21 & -7.40 \\
A4HYU2 & 3uiwA & 0.26 & 0.63 & 0.24 & -265.49 & -7.80 \\
A4I9V3 & 1ygyA & -0.31 & 0.79 & 0.27 & -632.91 & -8.41 \\
\hline
\end{tabular}

geometrical and their physicochemical properties. Ligands generally create favorable interactions with their binding sites; in this context, the active binding site of a hypothetical protein (UniProt ID: A4I8D6), which presented a drug score and a simple score of 0.81 and 0.62 , respectively, showed the best results (Table 2 ). To confirm these findings, a docking analysis was performed by using the SwissDock server, in which the FullFitness and Gibbs free energy $(\Delta G)$ parameters were evaluated. The results showed that strychnobiflavone showed affinity with a highest druggability score and a FullFitness of $-2985.25 \mathrm{kcal} / \mathrm{mol}$, besides an estimated $\Delta G$ of $-8.67 \mathrm{kcal} / \mathrm{mol}$ (Table 2 ). Since the sequence of this protein was annotated as a hypothetical protein, it was submitted to a functional annotation. In the results, this was identified as a hydrolase-like protein, with accuracy, sensitivity, and specificity values of $78.5 \%, 78.5 \%$, and $100 \%$, respectively.

3.4. Chemical-Protein Interactome Profile. The drug adverse reactions are undesirable, and since they can be caused by unexpected chemical-protein interactions, it is reasonable to predict interactions based on the mining of the chemicalprotein interactome (CPI) [53]. In this sense, DRAR-CPI and DDI-CPI servers were used to screen undesired interactions between strychnobiflavone and human proteins (Table 3), as well as between strychnobiflavone and other drugs (Table 4). The results showed that this molecule can interact with an alcohol dehydrogenase class-3 protein, whereas no interaction was found between this molecule and evaluated drugs.

\section{Discussion}

Flavonoids represent an important family of polyphenolic compounds that exist in plants, vegetables, and fruits. Since people use substantial amounts of these molecules daily, it is accepted that flavonoids are not toxic to humans [54]. Recently, a flavonoid derived from Strychnos pseudoquina stem bark, namely, strychnobiflavone, presented an effective antileishmanial activity against $L$. amazonensis and $L$. infantum promastigotes and amastigotes. In addition, the mechanism of action of this molecule in L. infantum was evaluated and proved to be related to alterations in the parasite's mitochondrial membrane $[19,20]$. In this context, the aim of the present study was to employ distinct bioinformatics programs to screen the metabolic pathway targeted by strychnobiflavone in L. infantum parasites.
The use of Leishmania promastigotes and amastigotes in in vitro studies to identify new antileishmanial products is still a key strategy in the development of new drugs [55]. However, it is not an easy task, since studies have shown the in vitro and/or in vivo biological action upon the parasites, but no mechanism of action has been proven. In this context, distinct bioinformatics strategies, such as target fishing and molecular docking assays, could be employed as technologies able to screen biological targets of distinct molecules in parasites, since they are based on the analysis of chemical structures by using information from biologically annotated databases, thus aiding many research groups [50].

Regarding the present study's results, changes in the parasite's metabolism were associated with three major enzymes related to the methylglyoxal degradation superpathway in L. infantum: Glyoxalase I, Glyoxalase II, and Aldo-keto reductase, which were evaluated by a STRING server. In this regard, the homology search performed among the selected sequences showed that six sequences presented significant differences between Leishmania and human proteins. Among them, a hypothetical protein (UniProt ID: A4I8D6), able to interact with Glyoxalase II, showed the highest druggability and molecular docking score and could be considered a possible molecular target for strychnobiflavone in L. infantum.

The methylglyoxal degradation superpathway has been also suggested to be a metabolic target of other chemotherapeutic agents against Plasmodium falciparum, Toxoplasma gondii, L. major, Trypanosoma brucei, Trypanosoma cruzi, Entamoeba histolytica, and Giardia lamblia [51, 56]. As a consequence, and due to the high similarity between Leishmania and Trypanosoma genus parasites, one could speculate that our computational approach was valid in identifying the possible biological target of strychnobiflavone in L. infantum.

Leishmania proteome information indicates that between $50 \%$ and $65 \%$ of all protein sequences have yet to be reported clearly [57] and are consequently classified as "uncharacterized or hypothetical" due to the fact that they present a low identity to known protein sequences [58]. The lack of identity with other sequenced organisms could be explained by the fact that, in the past, Leishmania was phylogenetically differentiated from the higher eukaryotes [59]. Thus, the hypothetical protein sequence identified here was submitted to an in silico functional annotation protocol, and results showed that it was predicted to be a hydroxylase-like protein. In this context, the data obtained in this study suggest the involvement of strychnobiflavone in the methylglyoxal 


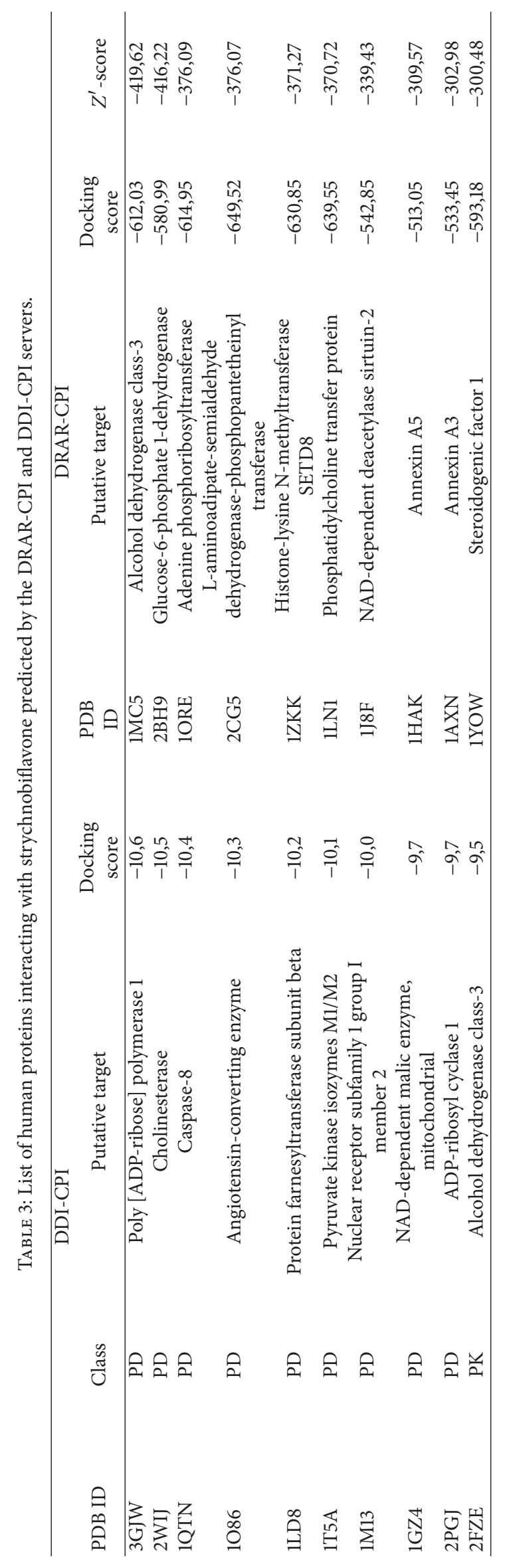


TABLE 4: List of drugs interacting with strychnobiflavone predicted by the DRAR-CPI and DDI-CPI serves.

\begin{tabular}{|c|c|c|c|c|}
\hline \multicolumn{2}{|c|}{ DDI-CPI } & \multicolumn{3}{|c|}{ DRAR-CPI } \\
\hline Library drug & Confidence & Library drug & Association score & $P$ value \\
\hline Betamethasone acetate & 1,0 & Dalfopristin 2 & 1,00 & 0,03 \\
\hline Buprenorphine hydrochloride & 1,0 & Cromoglicate 2 & $-1,00$ & 0,13 \\
\hline Candesartan cilexetil & 1,0 & Cisapride 6 & $-0,89$ & 0,06 \\
\hline Ceftriaxone 2 & 1,0 & Didanosine 2 & $-0,88$ & 0,03 \\
\hline Ciclesonide 2 & 1,0 & Darunavir 4 & $-0,84$ & 0,20 \\
\hline Clarithromycin 2 & 1,0 & Droperidol 2 & $-0,84$ & 0,03 \\
\hline Clarithromycin 3 & 1,0 & Aliskiren & 0,16 & 0,83 \\
\hline Dextromethorphan 3 & 1,0 & Indinavir 2 & 0,81 & 0,26 \\
\hline Dihydroergotamine 2 & 1,0 & Droperidol 3 & $-0,79$ & 0,11 \\
\hline Dihydroergotamine 3 & 1,0 & Folic acid 4 & $-0,78$ & 0,23 \\
\hline
\end{tabular}

degradation superpathway, due to its interaction with Glyoxalase II. In addition, the use of a CPI server, together with biology-based integrative systems, showed that no significant interaction with human proteins was found, then suggesting the absence of side effects if strychnobiflavone was used to treat human leishmaniasis.

In conclusion, it was proved strychnobiflavone interacts with the alcohol dehydrogenase class- 3 protein, and results showed that the putative metabolic pathway inhibited by the molecule in the parasites was the methylglyoxal degradation superpathway, with a hydrolase-like protein proving to be the molecular target in Leishmania. Due to similar findings in other trypanosomatids, it could be speculated that our strategy, using distinct bioinformatics tools, was valid and could be well employed to screen other biological targets evoked by distinct molecules in different pathogens. In addition, in vitro biological studies are currently under development to confirm our findings, and preliminary results have shown that strychnobiflavone does act on the methylglyoxal degradation superpathway in L. infantum.

\section{Conflicts of Interest}

The authors declare that they have no conflicts of interest.

\section{Authors' Contributions}

Eduardo Antonio Ferraz Coelho and Miguel Angel ChávezFumagalli conceived and designed the experiments. Miguel Angel Chávez-Fumagalli, Mônica Santos Schneider, Thaís Teodoro de Oliveira Santos, Daniela Pagliara Lage, Grasiele de Sousa Vieira Tavares, and Débora Vasconcelos Costa Mendonça performed the experiments. Miguel Angel Chávez-Fumagalli, Eduardo Antonio Ferraz Coelho, João Paulo Viana Leite, Ricardo Andrez Machado-de-Ávila, and Rodrigo Maia Pádua analyzed the data. Rodrigo Maia Pádua and Ricardo Andrez Machado-de-Ávila contributed reagents/materials/analysis tools. Eduardo Antonio Ferraz Coelho, Miguel Angel Chávez-Fumagalli, and Ricardo Andrez Machado-de-Ávila wrote the paper. In addition, all authors read and approved the final version of the manuscript.

\section{Acknowledgments}

This work was supported by grants from Pró-Reitoria de Pesquisa da Universidade Federal de Minas Gerais (Edital 02/2017), Instituto Nacional de Ciência e Tecnologia em Nanobiofarmacêutica (INCT NanoBiofar), FAPEMIG (CBB-APQ-00819-12 and CBB-APQ-01778-2014), and CNPq (APQ-482976/2012-8, APQ-488237/2013-0, and APQ467640/2014-9). Eduardo Antonio Ferraz Coelho is a grant recipient of CNPq. Miguel Angel Chávez-Fumagalli is a grant recipient of CAPES/FAPEMIG.

\section{References}

[1] P. D. Ready, "Epidemiology of visceral leishmaniasis," Journal of Clinical Epidemiology, vol. 6, no. 1, pp. 147-154, 2014.

[2] M. Maroli, M. D. Feliciangeli, L. Bichaud, R. N. Charrel, and L. Gradoni, "Phlebotomine sand flies and the spreading of leishmaniases and other diseases of public health concern," Medical and Veterinary Entomology, vol. 27, no. 2, pp. 123-147, 2013.

[3] G. Grimaldi Jr. and R. B. Tesh, "Leishmaniases of the New World: current concepts and implications for future research," Clinical Microbiology Reviews, vol. 6, no. 3, pp. 230-250, 1993.

[4] A. F. Frade, L. C. D. Oliveira, D. L. Costa et al., "TGFB1 and IL8 gene polymorphisms and susceptibility to visceral leishmaniasis," Infection, Genetics and Evolution, vol. 11, no. 5, pp. 912916, 2011.

[5] A. F. Druzian, A. S. D. Souza, D. N. D. Campos et al., "Risk factors for death from visceral leishmaniasis in an urban area of Brazil," PLOS Neglected Tropical Diseases, vol. 9, no. 8, article no. A041, 2015.

[6] A. Queiroz and N. V. Cavalcanti, "Risk factors for death in children with visceral leishmaniasis," PLOS Neglected Tropical Diseases, vol. 4, no. 11, p. e877, 2010.

[7] F. Frézard and C. Demicheli, "New delivery strategies for the old pentavalent antimonial drugs," Expert Opinion on Drug Delivery, vol. 7, no. 12, pp. 1343-1358, 2010.

[8] R. Herbrecht, S. Natarajan-Amé, Y. Nivoix, and V. Letscher-Bru, “The lipid formulations of amphotericin B," Expert Opinion on Pharmacotherapy, vol. 4, no. 8, pp. 1277-1287, 2003.

[9] S. S. Egger, S. Meier, C. Leu et al., "Drug interactions and adverse events associated with antimycotic drugs used for invasive aspergillosis in hematopoietic SCT," Bone Marrow Transplantation, vol. 45, no. 7, pp. 1197-1203, 2010. 
[10] M. A. Chávez-Fumagalli, T. G. Ribeiro, R. O. Castilho, S. Odília, A. Fernandes, and V. N. Cardoso, "New delivery systems for amphotericin B applied to the improvement of leishmaniasis treatment," Revista da Sociedade Brasileira de Medicina Tropical, vol. 48, no. 3, pp. 235-242, 2015.

[11] J. A. L. Lindoso, J. M. Costa, I. T. Queiroz, and H. Goto, "Review of the current treatments for leishmaniases," Research and Reports in Tropical Medicine, vol. 3, pp. 69-77, 2012.

[12] D. J. Newman, G. M. Cragg, and K. M. Snader, "Natural products as sources of new drugs over the period 1981-2002," Journal of Natural Products, vol. 66, no. 7, pp. 1022-1037, 2003.

[13] M. S. Butler, "Natural products to drugs: Natural product derived compounds in clinical trials," Natural Product Reports, vol. 22, no. 2, pp. 162-195, 2005.

[14] H. Hussain, A. Al-Harrasi, A. Al-Rawahi, I. R. Green, and S. Gibbons, "Fruitful decade for antileishmanial compounds from 2002 to late 2011," Chemical Reviews, vol. 114, no. 20, pp. 1036910428, 2014.

[15] T. G. Ribeiro, M. A. Chávez-Fumagalli, D. G. Valadares et al., "Antileishmanial activity and cytotoxicity of Brazilian plants," Experimental Parasitology emphasizes, vol. 143, no. 1, pp. 60-68, 2014.

[16] T. G. Ribeiro, A. M. Nascimento, B. O. Henriques et al., "Antileishmanial activity of standardized fractions of Stryphnodendron obovatum (Barbatimão) extract and constituent compounds," Journal of Ethnopharmacology, vol. 165, pp. 238-242, 2015.

[17] T. J. Schmidt, S. A. Khalid, A. J. Romanha et al., “The potential of secondary metabolites from plants as drugs or leads against protozoan neglected diseases-part I," Current Medicinal Chemistry, vol. 19, no. 14, pp. 2128-2175, 2012.

[18] A. Saklani and S. K. Kutty, "Plant-derived compounds in clinical trials," Drug Discovery Therapy, vol. 13, no. 3-4, pp. 161-171, 2008.

[19] P. S. Lage, P. H. R. Andrade, and A. S. Lopes, "Strychnos pseudoquina and its purified compounds present an effective in vitro antileishmanial activity," Evidence-Based Complementary and Alternative Medicine, vol. 2013, pp. 1-9, 2013.

[20] P. S. Lage, M. A. Chávez-Fumagalli, and J. T. Mesquita, "Antileishmanial activity and evaluation of the mechanism of action of strychnobiflavone flavonoid isolated from strychnos pseudoquina against leishmania infantum," Parasitology Research, vol. 114, no. 12, pp. 4625-4635, 2015.

[21] A. Cereto-Massagué, M. J. Ojeda, C. Valls, M. Mulero, G. Pujadas, and S. Garcia-Vallve, "Tools for in silico target fishing," Methods, vol. 71, pp. 98-103, 2015.

[22] J. C. Adams, M. J. Keiser, L. Basuino et al., "A mapping of drug space from the viewpoint of small molecule metabolism," PLoS Computational Biology, vol. 5, no. 8, Article ID e1000474, 2009.

[23] S. Kim, P. A. Thiessen, E. E. Bolton et al., "PubChem substance and compound databases," Nucleic Acids Research, vol. 44, no. 1, pp. D1202-D1213, 2016.

[24] L. Wang, C. Ma, P. Wipf, H. Liu, W. Su, and X.-Q. Xie, “Targethunter: An in silico target identification tool for predicting therapeutic potential of small organic molecules based on chemogenomic database," The AAPS Journal, vol. 15, no. 2, pp. 395-406, 2013.

[25] D. Gfeller, A. Grosdidier, M. Wirth, A. Daina, O. Michielin, and V. Zoete, "SwissTargetPrediction: A web server for target prediction of bioactive small molecules," Nucleic Acids Research, vol. 42, no. 1, pp. W32-W38, 2014.
[26] Z. Wang, L. Liang, Z. Yin, and J. Lin, "Improving chemical similarity ensemble approach in target prediction," Journal of Cheminformatics, vol. 8, no. 1, article no. 20, 2016.

[27] A. Lagunin, A. Stepanchikova, D. Filimonov, and V. Poroikov, "PASS: prediction of activity spectra for biologically active substances," Bioinformatics, vol. 16, no. 8, pp. 747-748, 2000.

[28] W. L. Devillé, F. Buntinx, L. M. Bouter et al., "Conducting systematic reviews of diagnostic studies: didactic guidelines," BMC Medical Research Methodology, vol. 2, no. 1, article 9, 2002.

[29] M. Kanehisa, S. Goto, Y. Sato, M. Furumichi, and M. Tanabe, "KEGG for integration and interpretation of large-scale molecular data sets," Nucleic Acids Research, vol. 40, no. 1, pp. D109D114, 2012.

[30] R. Caspi, R. Billington, L. Ferrer et al., "The MetaCyc database of metabolic pathways and enzymes and the BioCyc collection of pathway/genome databases," Nucleic Acids Research, vol. 44, no. 1, pp. D471-D480, 2016.

[31] D. Szklarczyk, A. Franceschini, S. Wyder et al., "STRING v10: protein-protein interaction networks, integrated over the tree of life," Nucleic Acids Research, vol. 43, pp. D447-D452, 2015.

[32] S. F. Altschul, W. Gish, W. Miller, E. W. Myers, and D. J. Lipman, "Basic local alignment search tool," Journal of Molecular Biology, vol. 215, no. 3, pp. 403-410, 1990.

[33] J. F. Collins, A. F. W. Coulson, and A. Lyall, "The significance of protein sequence similarities," Bioinformatics, vol. 4, no. 1, pp. 67-71, 1988.

[34] W. R. Pearson, "Comparison of methods for searching protein sequence databases," Protein Science, vol. 4, no. 6, pp. 1145-1160, 1995.

[35] W. R. Pearson, "Effective protein sequence comparison," Methods in Enzymology, vol. 266, pp. 227-258, 1996.

[36] J. Yang, R. Yan, A. Roy, D. Xu, J. Poisson, and Y. Zhang, "The I-TASSER Suite: protein structure and function prediction," Nature Methods, vol. 12, no. 1, pp. 7-8, 2015.

[37] A. Volkamer, D. Kuhn, F. Rippmann, and M. Rarey, "Dogsitescorer: A web server for automatic binding site prediction, analysis and druggability assessment," Bioinformatics, vol. 28, no. 15, Article ID bts310, pp. 2074-2075, 2012.

[38] A. Grosdidier, V. Zoete, and O. Michielin, "SwissDock, a protein-small molecule docking web service based on EADock DSS," Nucleic Acids Research, vol. 39, no. 2, pp. W270-W277, 2011.

[39] E. F. Pettersen, T. D. Goddard, C. C. Huang et al., "UCSF Chimera-a visualization system for exploratory research and analysis," Journal of Computational Chemistry, vol. 25, no. 13, pp. 1605-1612, 2004.

[40] M. A. Chávez-Fumagalli, M. S. Schneider, D. P. Lage, R. A. Machado-de-Ávila, and E. A. F. Coelho, "An in silico functional annotation and screening of potential drug targets derived from Leishmania spp. hypothetical proteins identified by immunoproteomics," Experimental Parasitology emphasizes, vol. 176, pp. 66-74, 2017.

[41] R. D. Finn, P. Coggill, R. Y. Eberhardt et al., "The Pfam protein families database: towards a more sustainable future," Nucleic Acids Research, vol. 44, no. 1, pp. D279-D285, 2016.

[42] H. Mi, S. Poudel, A. Muruganujan, J. T. Casagrande, and P. D. Thomas, "PANTHER version 10: expanded protein families and functions, and analysis tools," Nucleic Acids Research, vol. 44, no. D1, pp. D336-D342, 2016.

[43] D. Wilson, R. Pethica, Y. Zhou et al., "SUPERFAMILY Sophisticated comparative genomics, data mining, visualization 
and phylogeny," Nucleic Acids Research, vol. 37, no. 1, pp. D380D386, 2009.

[44] A. Y. Lee, C. S. Lee, and R. N. Van Gelder, "Scalable metagenomics alignment research tool (SMART): A scalable, rapid, and complete search heuristic for the classification of metagenomic sequences from complex sequence populations," BMC Bioinformatics, vol. 17, no. 1, article no. 292, 2016.

[45] I. Sillitoe, T. E. Lewis, A. Cuff et al., "CATH: Comprehensive structural and functional annotations for genome sequences," Nucleic Acids Research, vol. 43, no. 1, pp. D376-D381, 2015.

[46] N. Rappoport, S. Karsenty, A. Stern, N. Linial, and M. Linial, "ProtoNet 6.0: Organizing 10 million protein sequences in a compact hierarchical family tree," Nucleic Acids Research, vol. 40, no. 1, pp. D313-D320, 2012.

[47] L. Yang, K. J. Wang, L. S. Wang et al., "Chemical-protein interactome and its application in off-target identification," Interdisciplinary Sciences: Computational Life Sciences, vol. 3, no. 1, pp. 22-30, 2011.

[48] H. Luo, J. Chen, L. Shi et al., "DRAR-CPI: a server for identifying drug repositioning potential and adverse drug reactions via the chemical-protein interactome," Nucleic Acids Research, vol. 39, supplement 2, pp. W492-W498, 2011.

[49] H. Luo, P. Zhang, H. Huang et al., "DDI-CPI, a server that predicts drug-drug interactions through implementing the chemical-protein interactome," Nucleic Acids Research, vol. 42, no. 1, pp. W46-W52, 2014.

[50] J. L. Jenkins, A. Bender, and J. W. Davies, "In silico target fishing: predicting biological targets from chemical structure," Drug Discovery Today: Technologies, vol. 3, no. 4, pp. 413-421, 2006.

[51] M. Sousa Silva, A. E. N. Ferreira, R. Gomes, A. M. Tomás, A. Ponces Freire, and C. Cordeiro, "The glyoxalase pathway in protozoan parasites," International Journal of Medical Microbiology, vol. 302, no. 4-5, pp. 225-229, 2012.

[52] S. C. Bull and A. J. Doig, "Properties of protein drug target classes," PLoS ONE, vol. 10, no. 3, Article ID e0117955, 2015.

[53] A. Lavecchia and C. Cerchia, "In silico methods to address polypharmacology: Current status, applications and future perspectives," Drug Discovery Therapy, vol. 21, no. 2, pp. 288298, 2016.

[54] I. L. K. Wong, K.-F. Chan, T. H. Chan, and L. M. C. Chow, "Flavonoid dimers as novel, potent antileishmanial agents," Journal of Medicinal Chemistry, vol. 55, no. 20, pp. 8891-8902, 2012.

[55] C. P. Hart, "Finding the target after screening the phenotype," Drug Discovery Therapy, vol. 10, no. 7, pp. 513-519, 2005.

[56] S. Wyllie and A. H. Fairlamb, "Methylglyoxal metabolism in trypanosomes and leishmania," Seminars in Cell \& Developmental Biology, vol. 22, no. 3, pp. 271-277, 2011.

[57] P. Cuervo, G. B. Domont, and J. B. De Jesus, "Proteomics of trypanosomatids of human medical importance," Journal of Proteomics, vol. 73, no. 5, pp. 845-867, 2010.

[58] G. Lubec, L. Afjehi-Sadat, J.-W. Yang, and J. P. P. John, "Searching for hypothetical proteins: Theory and practice based upon original data and literature," Progress in Neurobiology, vol. 77, no. 1-2, pp. 90-127, 2005.

[59] A. P. Jackson, "Genome evolution in trypanosomatid parasites," Parasitology, pp. 1-17, 2014. 


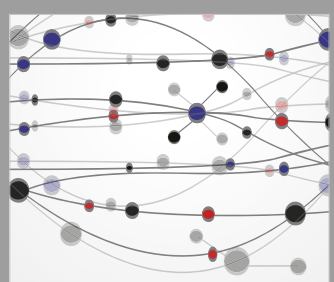

The Scientific World Journal
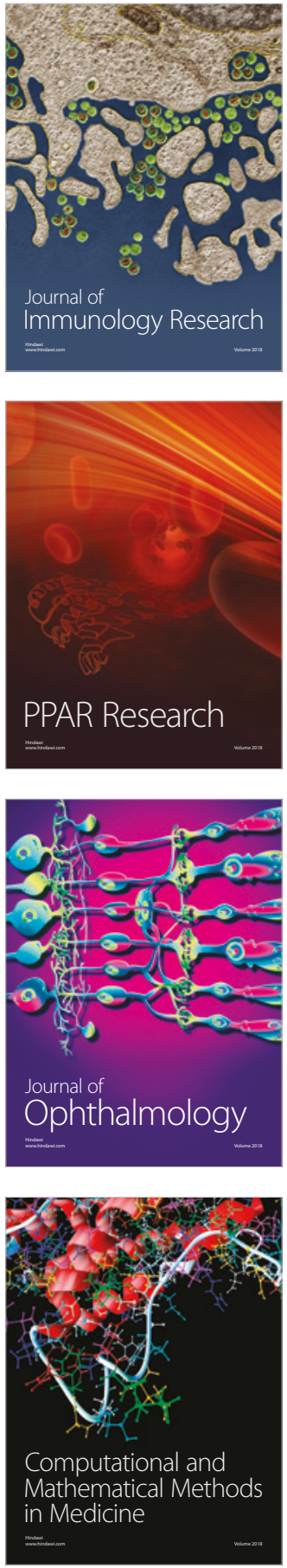

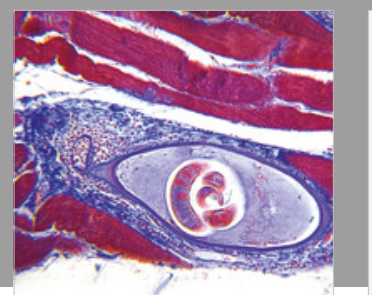

Gastroenterology Research and Practice

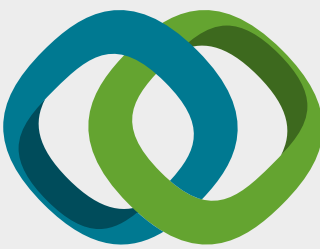

\section{Hindawi}

Submit your manuscripts at

www.hindawi.com
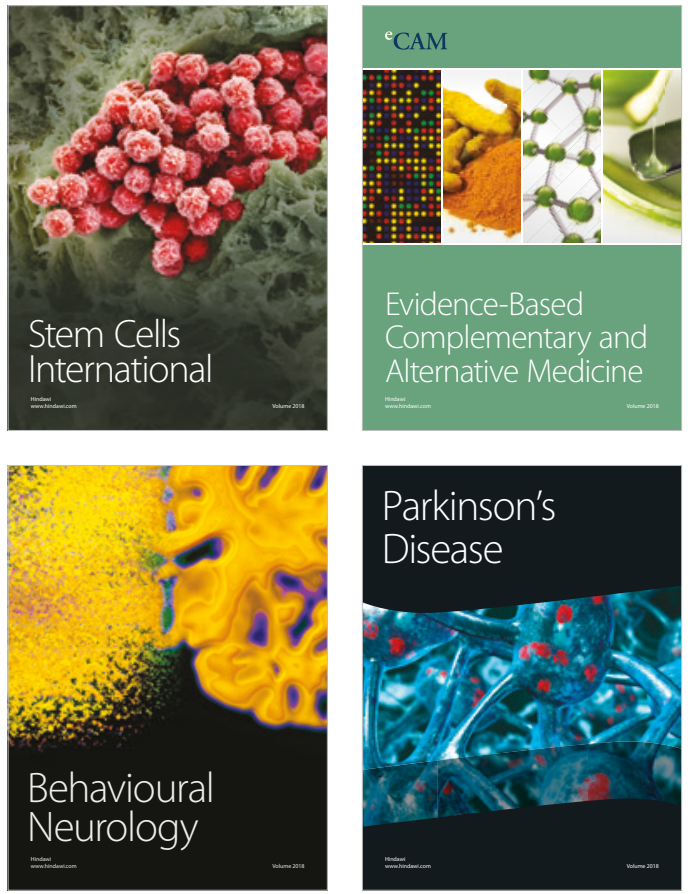

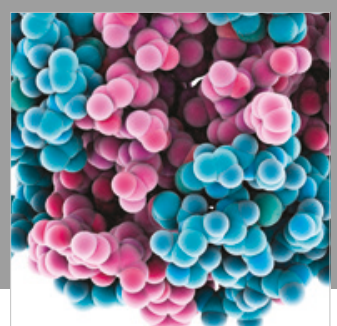

ournal of

Diabetes Research

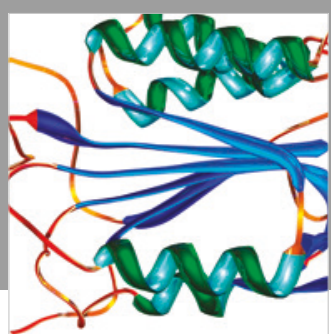

Disease Markers
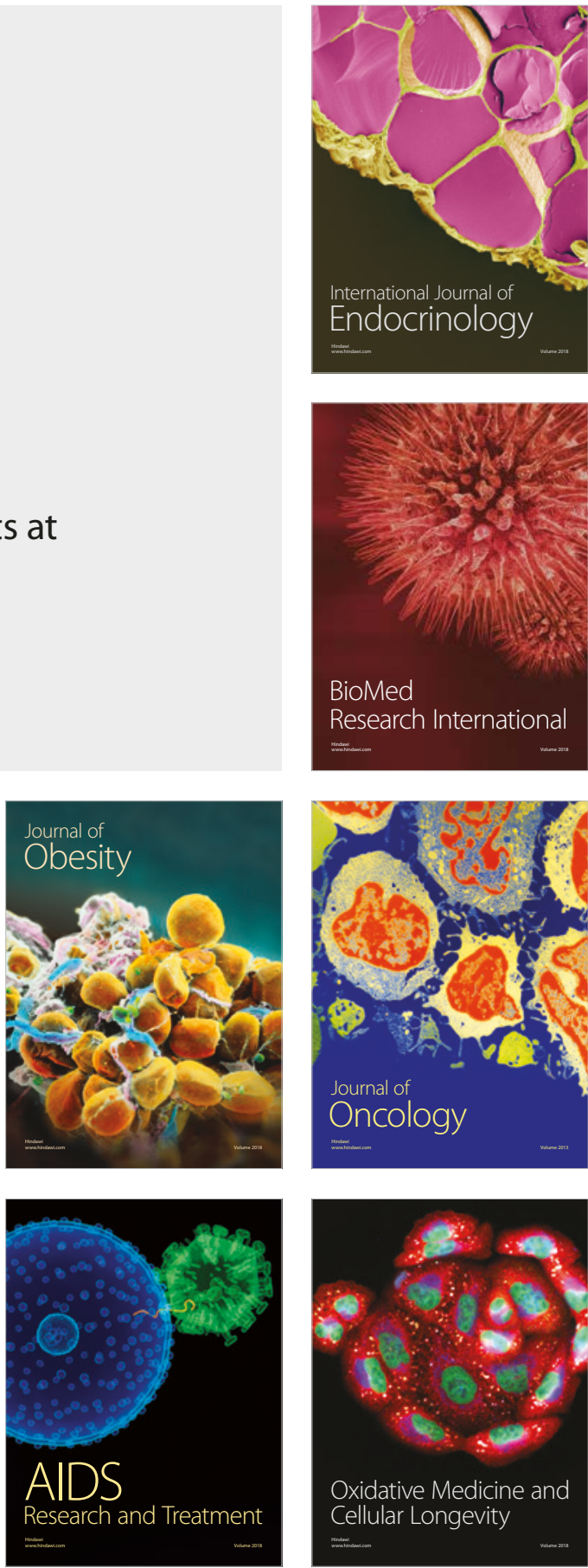